\title{
Edge Preserving Based on Weighted Guided Image Filtering
}

\author{
Dr. D.Vishnu Vardhan M.Tech, Phd ${ }^{1}$, Bandaru Bhavana ${ }^{2}$, Dr. C. Nagaraju ${ }^{3}$ \\ ${ }^{1}$ Assistant Professor, Department of ECE, JNTUA College of Pulivendula \\ ${ }^{2}$ II M. Tech student, Department of ECE, JNTUA College of Pulivendula \\ ${ }^{3}$ Associate Professor, Department of CSE, YSR Engineering College of YVU
}

\begin{abstract}
In this paper a weighted guided image filter (WGIF) is being proposed incorporating an edge-aware weighting technique into the guided image filter (GIF). The WGIF preserves sharp edges as well as existing global filters, and the intricacy of the WGIF is $O(N)$ for an image with $N$ pixels which is almost the same as the GIF. As WGIF is simple, it has numerous applications in the fields of computational photography and image processing. The WGIF provides advantages of both local and global smoothing filters in the sense that: 1) the complexity of the WGIF is $O(N)$ for an image with $N$ pixels, which is similar as the GIF and 2) the WGIF can avoid halo artifacts like the existing global smoothing filters. The WGIF is practical for single image feature enhancement. Finally, the parameters like mean, standard deviation, mean square error and PSNR for GIF and WGIF techniques are compared.
\end{abstract}

Keywords: Weighted guided image filter, edge aware technique, global and local filters, image enhancement

\section{Introduction}

In human visual perception, edges provide an effective and expressive stimulation which is important for neural interpretation of a scene. In the fields of image processing and in many computational photography employ smoothing techniques which could preserve edges better. In smoothing process an image to be filtered is typically decomposed into two layers: a base layer composed by homogeneous regions with sharp edges and a detail layer formed by either noise, e.g., a random pattern with zero mean, or texture, e.g, a repeated pattern with usual arrangement. There are two types of edge-preserving image smoothing techniques: global filters such as the weighted least squares (WLS) [8] filter and local filters such as bilateral filter (BF) [3], trilateral filter, and their accelerated versions [4], as well as guided image filter (GIF) [11].Though the global optimization based filters frequently yield excellent quality, they have high computational cost. Comparing with the global optimization based filters [1], [2], [8] and [9], the local filters are generally simpler. However, the local filters cannot conserve sharp edges like the global optimization based filters.

Halo artifacts were usually produced by the local filters when they were adopted to smooth edges. Major reason that the BF/GIF produces halo artifacts was both spatial similarity parameter and range similarity parameter in the BF [3] were fixed. But both the spatial similarity and the range similarity parameters of the $\mathrm{BF}$ could be adaptive to the content of the image to be filtered. Unfortunately as pointed out in [6], problem with adaptation of the parameters will destroy the 3D convolution form in [5]. We introduce in present paper, an edge-aware weighting technique and incorporated into the GIF to form a weighted GIF (WGIF). Local variance in $3 \times 3$ window of pixel in a guidance image is applied to calculate the edge-aware weighting. The local variance of a pixel is normalized by the local variance of all pixels in guidance image. The normalized weighting is then adopted to design the WGIF. As a result, halo artifacts can be avoided by using the WGIF. Similar to the GIF in [12], the WGIF also avoids gradient reversal. In addition, the intricacy of the WGIF is $\mathrm{O}(\mathrm{N})$ for an image with $\mathrm{N}$ pixels which is the same as that of the GIF [14]. These features allow many applications of the WGIF for single image detail enhancement, single image mist removal, and fusion of differently exposed images.

\section{Edge Preserving Smoothing Techniques}

The task of edge-preserving smoothing is to crumble an image $\mathrm{X}$ into two parts as follows:

$$
X(p)=\hat{J}(p)+e(p)
$$

where $\hat{\mathrm{J}}$ is a reconstructed image formed by uniform regions with sharp edges, e is noise or texture, and $\mathrm{p}(=(\mathrm{x}, \mathrm{y}))$ is a position. $\hat{\mathrm{J}}$ and $\mathrm{e}$ are called base layer and detail layer, respectively. One of edge-preserving smoothing techniques is based on local filtering. Bilateral filter( BF) [3] is widely used due to its simplicity but suffer from "gradient reversal" artifacts usually observed in detail enhancement of conventional LDR images. Then GIF [14] was introduced to overcome this problem. In this GIF, a guidance image $G$ was used which could be similar to the image $\mathrm{X}$ which is to be filtered. $\hat{J}$ is a linear transform of $G$ in the window $\Omega \varsigma$ $\left(p^{\prime}\right)$.To determine the linear coefficients $\left(a p^{\prime}, b p^{\prime}\right)$, a constraint is added to $\mathrm{X}$ and $\hat{\mathrm{J}}$ as in Equation (1). The values of $\mathrm{ap}^{\prime}$ and $\mathrm{bp}^{\prime}$ are then obtained by minimizing a cost function $\mathrm{E}\left(\mathrm{ap} \mathrm{p}^{\prime}, \mathrm{bp} \mathrm{p}^{\prime}\right)$ which is defined as

$$
\mathrm{E}=\sum_{P € \Omega \varsigma}\left[\left(\mathrm{ap}^{\prime} \mathrm{G}(\mathrm{p})+\mathrm{bp}^{\prime}-\mathrm{X}(\mathrm{p})\right)^{\wedge} 2+\lambda \mathrm{ap} \mathrm{p}^{\wedge} 2\right]
$$

where $\lambda$ is a regularization parameter.

Another type of edge-preserving smoothing techniques was based on global optimization. The Weighted Least Square filter [8] was a typical example and it was derived by minimizing the following quadratic cost function:

$$
\mathrm{E}=\sum_{p=1}^{N}\left[(\hat{\mathrm{J}}(\mathrm{p})-\mathrm{X}(\mathrm{p}))^{2}+\lambda(\mathrm{\phi})\|\nabla \hat{\mathrm{J}}(p)\|^{2}\right]
$$




\section{International Journal of Science and Research (IJSR) \\ ISSN (Online): 2319-7064 \\ Index Copernicus Value (2013): 6.14 | Impact Factor (2015): 6.391}

where $\mathrm{N}$ is the total number of pixels in an image. The two major differences between the WLS filter and the GIF. 1) The GIF [14] is based on local optimization while the WLS filter in based on global optimization. As such, the difficulty of the GIF is $\mathrm{O}(\mathrm{N})$ for an image with $\mathrm{N}$ number of pixels and the Weighted Least Square filter [8] is more complicated than the GIF. 2) The value of $\lambda$ is fixed in the GIF while it is adaptive to local gradients in the WLS filter [8]. One possible problem for the GIF [14] is halos which could be reduced by the WLS filter. The spatial varying image gradients aware weighting $\lambda x(p)$ and $\lambda y(p)$ are very important for the WLS filter to avoid halo artifacts.

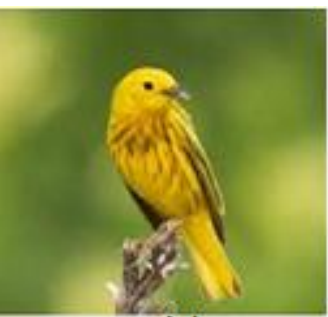

(a)

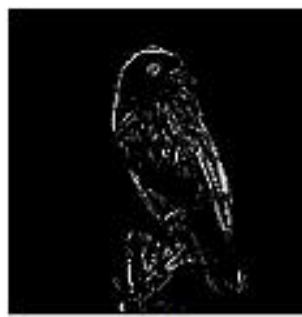

(b)
Figure: a) Input image b) edge of input image

\section{Existing Methods}

\section{Bilateral Filter}

The bilateral filter was perhaps the simplest which computed the filtering output at each pixel as the average of near-by pixels, weighted by the Gaussian of both range and spatial distance. The bilateral filter [3] smooth's the image while preserving edges. Constraint of the bilateral filter was it endure from "gradient reversal" artifacts. The reason was that when a pixel (often on an edge) has few similar pixels around it, the Gaussian weighted average is unstable. Efficiency was another problem regarding the bilateral filter.

\section{Non-average Filter}

Edge-preserving filtering could also be achieved by nonaverage filters. The median filter was a familiar edge-aware operator, and was a special case of local histogram filters. Histogram filters had $\mathrm{O}(\mathrm{N})$ time implementations in a way as the bilateral grid. The non-average filters were often computationally expensive.

\section{Guided Image Filter}

A general linear translation-variant filtering process, which involved a guidance image $I$, an filtering input image $p$, and an output image q. The filtering output at a pixel I was expressed as a weighted average:

$$
q i=\sum_{j} \mathrm{Wij}(I) p j
$$

where $\mathrm{i}$ and $\mathrm{j}$ were pixel indexes. The filter kernel Wij was a function of the guidance image I and independent of $\mathrm{p}$. This filter was linear with respect to $\mathrm{p}$. The guided filter was a local linear model between the guidance I and the filtering output q. We assumed that $\mathrm{q}$ was a linear transform of I in a window ! $\mathrm{k}$ centered at the pixel $\mathrm{k}$ :

$$
\mathrm{qi}=\mathrm{akIi}+\mathrm{bk}, \forall \mathrm{i} € \mathrm{wk}
$$

where (ak,bk) were some linear coefficients assumed to be constant in wk. A square window of a radius $r$ was used. This local linear model ensures that $q$ has an edge only if I had an edge, because $\boldsymbol{\nabla q}=\mathrm{a} \nabla \mathrm{I}$. The output q was modeled as the input $\mathrm{p}$ subtracting some unwanted components $\mathrm{n}$ like noise/textures:

$$
\mathrm{qi}=\mathrm{pi}-\mathrm{ni} .
$$

\section{Adaptive Bilateral Filter}

Both range similarity parameter and spatial similarity parameter were adaptive to the content of filtered image [4]. However, adaptation of the parameters destroyed the 3-D convolution form. It was time consuming to extract fine details from a set of differently exposed images by the content adaptive bilateral filters because each input image needed to be decomposed individually. A content adaptive bilateral filter [13] was proposed in gradient domain by taking the characteristics of the human visual systems into consideration. The proposed bilateral filter could be applied to extract fine details from a set of images simultaneously. Similar to the content adaptive bilateral filters the acceleration of the proposed filter could be an issue. Fortunately, the idea in might be borrowed to accelerate the proposed filter.

\section{Adaptive Guided Image Filter}

An adaptive guided image filtering (AGF) [10] able to perform halo-free edge slope enhancement and noise reduction simultaneously. The intensity range domain of BLF and kernel function of GIF were similar in principle, because each of them takes the intensity value of center pixel $\mathrm{p}$, local neighbors $\mathrm{q}$ and a smoothing parameter ( $\sigma \mathrm{r}$ in $\mathrm{BLF}, \varepsilon$ in GIF) in the computation process. This was based on the shifting technique of $\mathrm{ABF}$, in which the offset $\xi \mathrm{p}$ was added to the intensity value of center pixel pin the intensity range domain of BLF. The same strategy was applied to AGF - the offset is added to the intensity value of center pixel pin the kernel weights function of GIF.

$$
\operatorname{AGF}(\mathrm{I}) \mathrm{p}=\sum_{\mathrm{q} \epsilon \omega \mathrm{k}} \text { WAGFpq(G)Iq }
$$

It was effective to remove noise and sharpens the edges simultaneously, without producing overshoot and undershoot artifacts as the ideal approach. Disadvantage of AGF in terms of computation cost, where the computational complexity was $\mathrm{O}(\mathrm{N})$ compared to $\mathrm{O}(|\mathrm{w}| 2)$ of $\mathrm{ABF}$.

\section{Proposed Method}

The key hypothesis of the WGIF is a confined linear model between the guidance image $G$ and the filtering output $\hat{J}$. The model ensures that the output $\hat{J}$ has an edge only if the guidance image $\mathrm{G}$ has an edge. Let $\mathrm{G}$ be a guidance image and $\sigma^{2\left(P^{\prime}\right)}$ be the variance of $\mathrm{G}$. An edge-aware weighting $\Upsilon \mathrm{G}\left(\mathrm{P}^{\prime}\right)$ is defined by using local variances of $3 \times 3$ windows of all pixels as follows:

$$
\Upsilon G\left(P^{\prime}\right)=\left(\frac{1}{N}\right) \sum_{P=1}^{N} \frac{\left.\llbracket(\sigma)^{2} G\left(P^{\prime}\right)+\varepsilon\right)}{\sigma^{2} G(P)+\varepsilon}
$$

where $\varepsilon$ is a small constant and its value is selected as $(0.001 \times \mathrm{L})^{\wedge} 2$ while $\mathrm{L}$ is the dynamic range of the input image. All the pixels in the guidance image are used in the computation of $\mathrm{rG}\left(\mathrm{P}^{\prime}\right)$. In addition, the weighting $\mathrm{rG}\left(\mathrm{P}^{\prime}\right)$ measures the importance of pixel $\mathrm{P}^{\prime}$ with respect to the whole guidance image. The value of $\mathrm{rG}\left(\mathrm{P}^{\prime}\right)$ is usually larger than 1 if $\mathrm{p}^{\prime}$ is at an edge and smaller than 1 if $\mathrm{p}^{\prime}$ is in a flat area. To prevent feasible blocking artifacts from appearing 


\section{International Journal of Science and Research (IJSR) \\ ISSN (Online): 2319-7064}

Index Copernicus Value (2013): 6.14 | Impact Factor (2015): 6.391

in the concluding image, the value of $\Upsilon \mathrm{G}\left(\mathrm{P}^{\prime}\right)$ is smoothed by a Gaussian filter. The proposed weighting $\mathrm{rG}\left(\mathrm{P}^{\prime}\right)$ is incorporated into the cost function $\mathrm{E}\left(\mathrm{ap}^{\prime}, \mathrm{bp}^{\prime}\right)$. As such, the elucidation is obtained by minimizing the difference between the image to be filtered $\mathrm{X}$ and the filtering output $\hat{\mathrm{J}}$

$$
E=\sum_{P \in \Omega \varsigma} \llbracket\left[\left(a p^{\prime} G(p)+b p^{\prime}-\rrbracket X(p)\right)^{2}+\left(\frac{\lambda}{r G\left(p^{\prime}\right)}\right) a p^{\prime 2}\right]
$$

The optimal values of $\mathrm{ap}^{\prime}$ and $\mathrm{bp}^{\prime}$ are computed as

$$
\begin{gathered}
\mathrm{ap}^{\prime}=\frac{\mu \mathrm{G} \bigcirc \mathrm{X}, \varsigma 1\left(\mathrm{P}^{\prime}\right)-\mu \mathrm{G} \cdot \varsigma 1\left(\mathrm{P}^{\prime}\right) \mu \mathrm{X}, \varsigma 1\left(\mathrm{P}^{\prime}\right)}{\sigma 2 \mathrm{G}, \varsigma 1\left(\mathrm{P}^{\prime}\right)+\left(\frac{\lambda}{\Upsilon \mathrm{G}\left(\mathrm{P}^{\prime}\right)}\right)} \\
\mathrm{bp}^{\prime}=\mu \mathrm{X}, \varsigma 1\left(\mathrm{P}^{\prime}\right)-\mathrm{ap}^{\prime} \mu \mathrm{G}, \varsigma 1\left(\mathrm{P}^{\prime}\right)
\end{gathered}
$$

where $\bigcirc$ is the element-by-element product of two matrices. $\mu G \bigcirc X, \varsigma 1\left(\mathrm{P}^{\prime}\right), \mu \mathrm{G} . \varsigma 1\left(\mathrm{P}^{\prime}\right)$ and $\mu X, \varsigma 1\left(P^{\prime}\right)$ are the mean values of $\mathrm{G} \bigcirc \mathrm{X}, \mathrm{G}$ and $\mathrm{X}$, respectively. The final value of $\hat{J}(p)$ is given as follows:

$$
\hat{\mathrm{J}}(\mathrm{p})=\operatorname{apG}(\mathrm{P})+\mathrm{bp}
$$

where ap and $b p$ are mean values of $a p^{\prime}$ and $b p^{\prime}$. For easy analysis, the images $X$ and $G$ are assumed to be the same. Consider the case that the pixel $\mathrm{p}^{\prime}$ is at an edge. The value of $\mathrm{rX}\left(\mathrm{p}^{\prime}\right)$ is usually much larger than $1 . \mathrm{pp}^{\prime}$ in the WGIF is closer to 1 than $\mathrm{ap}^{\prime}$ in the GIF [14]. This implies that sharp edges are potted better by the WGIF than the GIF [14].

\section{Experiment Results}

This method has been tested on different images and compared the parameters like mean, standard deviation, mean square error and PSNR.

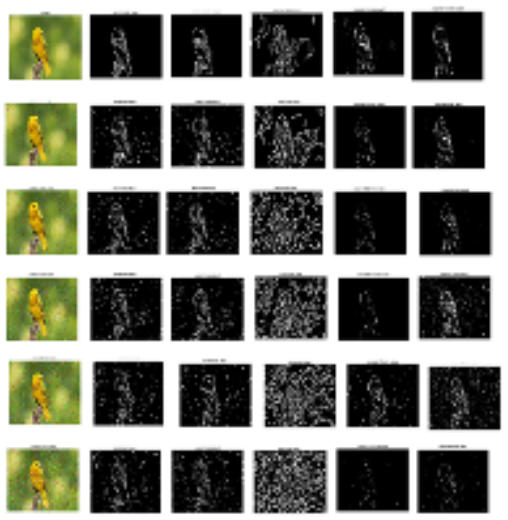

Figure: Output figures of Sobel, Prewitt, Canny, GIF and Proposed method with addition of noise
Table: Comparison of MEAN of existing and proposed method

\begin{tabular}{|c|c|c|c|c|c|}
\hline MEAN & SOBEL & PREWITT & CANNY & GIF & WGIF \\
\hline Noise 0.01 & 0.0116 & 0.0100 & 0.0554 & 0.0232 & 0.0018 \\
\hline Noise 0.02 & 0.0103 & 0.0094 & 0.0780 & 0.0231 & 0.0018 \\
\hline Noise 0.03 & 0.0116 & 0.0120 & 0.1036 & 0.0235 & 0.0034 \\
\hline Noise 0.04 & 0.0099 & 0.0092 & 0.1280 & 0.0237 & 0.0062 \\
\hline Noise 0.05 & 0.0085 & 0.0077 & 0.1302 & 0.0237 & 0.0073 \\
\hline
\end{tabular}

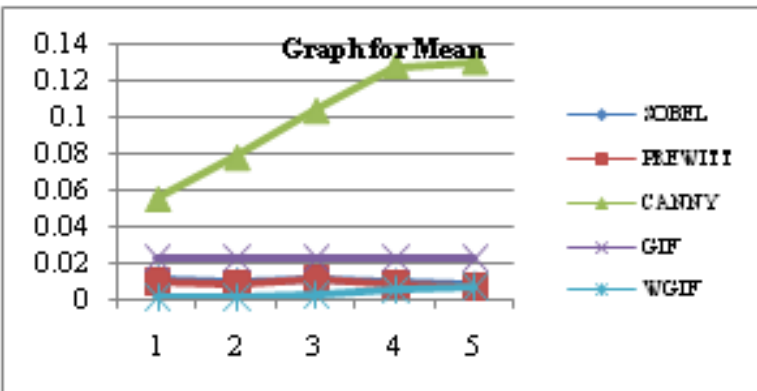

Graph: Comparing MEAN of existing and proposed method

Table: Comparison of STANDARD DEVIATION of existing and proposed method

\begin{tabular}{|c|c|c|c|c|c|}
\hline STD DEV & SOBEL & PREWITT & CANNY & GIF & WGIF \\
\hline Noise 0.01 & 0.0668 & 0.0838 & 0.0776 & 0.0878 & 0.0621 \\
\hline Noise 0.02 & 0.0726 & 0.0964 & 0.0817 & 0.0875 & 0.0686 \\
\hline Noise 0.03 & 0.0823 & 0.0885 & 0.0872 & 0.0885 & 0.0777 \\
\hline Noise 0.04 & 0.0846 & 0.0882 & 0.0863 & 0.0887 & 0.0800 \\
\hline Noise 0.05 & 0.0920 & 0.0994 & 0.0950 & 0.0880 & 0.0828 \\
\hline
\end{tabular}

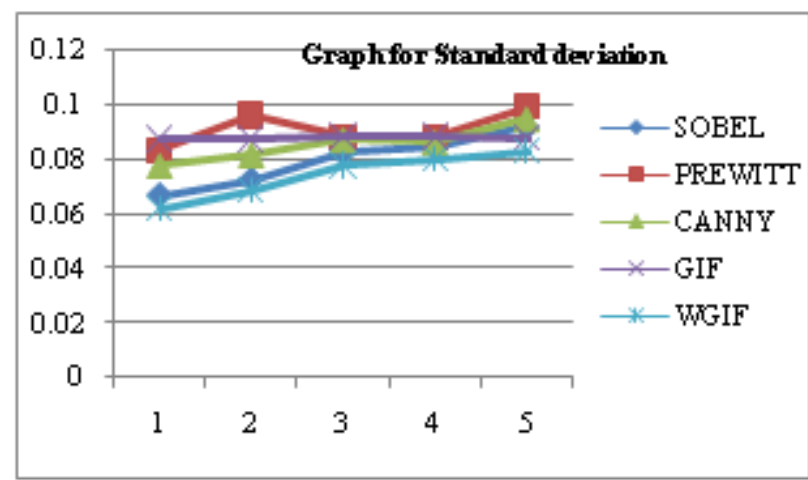

Graph: Comparing STANDARD DEVIATION of existing and proposed method

Table: Comparison of MEAN SQUARE ERROR of existing and proposed method

\begin{tabular}{|c|c|c|c|c|c|}
\hline MSE & SOBEL & PREWITT & CANNY & GIF & WGIF \\
\hline Noise 0.01 & $2.8940 \mathrm{e}+03$ & $3.5314 \mathrm{e}+03$ & $1.1309 \mathrm{e}+04$ & $1.2210 \mathrm{e}+04$ & $2.3441 \mathrm{e}+03$ \\
\hline Noise 0.02 & $6.9231 \mathrm{e}+03$ & $7.2634 \mathrm{e}+03$ & $1.7524 \mathrm{e}+04$ & $1.3859 \mathrm{e}+04$ & $5.1809 \mathrm{e}+03$ \\
\hline Noise 0.03 & $1.2106 \mathrm{e}+04$ & $1.2638 \mathrm{e}+04$ & $2.1199 \mathrm{e}+04$ & $1.4736 \mathrm{e}+04$ & $8.0844 \mathrm{e}+03$ \\
\hline Noise 0.04 & $1.4259 \mathrm{e}+04$ & $1.4929 \mathrm{e}+04$ & $2.1800 \mathrm{e}+04$ & $1.555 \mathrm{e}+04$ & $1.1131 \mathrm{e}+04$ \\
\hline Noise 0.05 & $1.6811 \mathrm{e}+04$ & $1.7295 \mathrm{e}+04$ & $2.4209 \mathrm{e}+04$ & $1.6291 \mathrm{e}+04$ & $1.2750 \mathrm{e}+04$ \\
\hline
\end{tabular}

Volume 5 Issue 6, June 2016 www.ijsr.net 


\section{International Journal of Science and Research (IJSR) \\ ISSN (Online): 2319-7064}

Index Copernicus Value (2013): 6.14 | Impact Factor (2015): 6.391

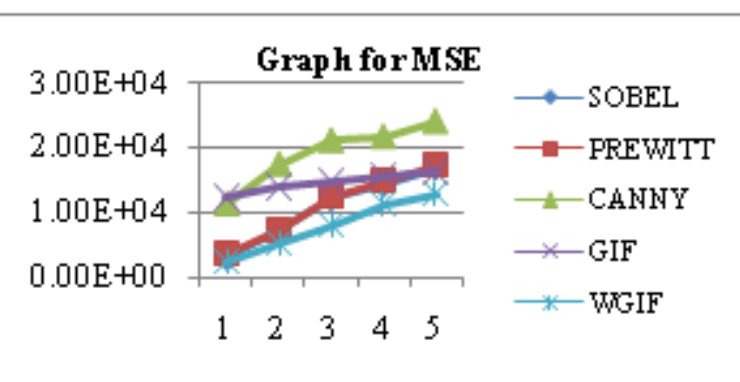

Graph: Comparing MEAN SQUARE ERROR of existing and proposed method

Table: Comparison of PSNR of existing and proposed method

\begin{tabular}{|c|c|c|c|c|c|}
\hline PSNR & SOBEL & PREWITT & CANNY & GIF & WGIF \\
\hline Noise 0.01 & 13.5497 & 12.6853 & 7.6307 & 7.2975 & 14.4650 \\
\hline Noise 0.02 & 9.7618 & 9.5534 & 5.7286 & 6.7475 & 11.0208 \\
\hline Noise 0.03 & 7.3349 & 7.1480 & 4.9017 & 6.4809 & 9.0883 \\
\hline Noise 0.04 & 6.6238 & 6.4244 & 4.7802 & 6.3170 & 7.6995 \\
\hline Noise 0.05 & 5.9089 & 5.7857 & 4.3251 & 6.0454 & 7.1097 \\
\hline
\end{tabular}

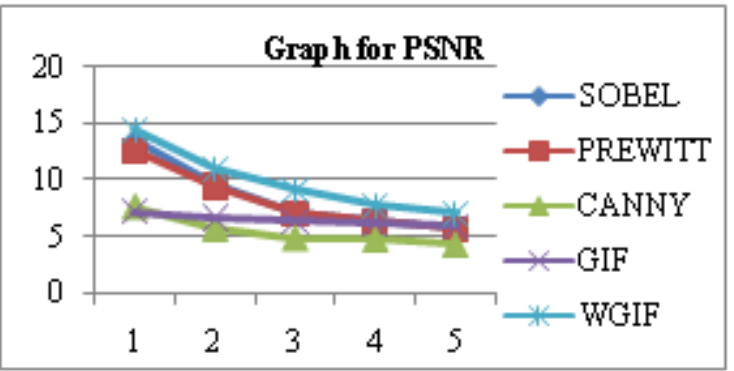

Graph: Comparing PSNR of existing and proposed method

From the experimental results, we can show that proposed method i..e, Weighted Guided Image Filter is better approach.

CASE 1 The first parameter we consider is MEAN which when we compare with all existing methods by plotting a graph we can see that proposed method has least mean.

CASE 2 The second parameter we consider is Standard deviation which when we compare with all existing methods by plotting a graph we can see that proposed method has least standard deviation.

CASE 3 The third parameter we consider is Mean square error which when we compare with all existing methods by plotting a graph we can see that proposed method has least mean square error.

CASE 4 The last parameter we consider is PSNR which when we compare with all existing methods by plotting a graph we can see that proposed method has highest PSNR.

\section{Conclusion and Discussion}

This paper describes weighted guided image filter (WGIF) by incorporating an edge-aware weighting into the guided image filter (GIF). The WGIF conserve sharp edges as well as existing global filters, and the difficulty of the WGIF is $\mathrm{O}(\mathrm{N})$ for an image with $\mathrm{N}$ pixels which is almost the same as the GIF. Due to the simplicity of the WGIF, it has numerous applications in the fields of computational photography and image processing. Experimental results show that the ensuing algorithms can generate images with excellent visual quality as those of global filters.
WGIF can also be adopted to improve the anisotropic diffusion, Poisson image editing, etc. All these research problems will be studied in future research.

\section{References}

[1] L. I. Rudin, S. Osher, and E. Fatemi, "Nonlinear total variation based noise removal algorithms," Phys. D, Nonlinear Phenomena, vol. 60, nos. 1-4, pp. 259-268, Nov. 1992.

[2] P. Charbonnier, L. Blanc-Feraud, G.Aubert, and M. Barlaud, "Deterministic edge-preserving regularization in computed imaging," IEEE Trans. Image Process., vol. 6, no. 2, pp. 298-311, Feb. 1997.

[3] C. Tomasi and R. Manduchi, "Bilateral filtering for gray and color images," in Proc. IEEE Int. Conf. Comput. Vis., Jan. 1998, pp. 836-846.

[4] F. Durand and J. Dorsey, "Fast bilateral filtering for the display of high dynamic-range images, "ACM Trans. Graph., vol. 21, no. 3, pp. 257-266,nAug. 2002.

[5] J. Chen, S. Paris, and F. Durand, "Real-time edge-aware image processing with the bilateral grid," ACM Trans. Graph., vol. 26, no. 3, pp. 103-111, Aug. 2007

[6] B. Y. Zhang and J. P. Allebach, "Adaptive bilateral filter for sharpness enhancement and noise removal, "IEEE Trans. Image Process., vol. 17, no. 5, pp. 664678, May 2008.

[7] J. Kopf et al., "Deep photo: Model-based photograph enhancement and viewing," inProc. SIGGRAPH Asia, 2008, pp. 1-10.

[8] Z. Farbman, R. Fattal, D. Lischinski, and R. Szeliski, "Edge-preserving decompositions for multi-scale tone and detail manipulation," ACM Trans. Graph., vol. 27, no. 3, pp. 249-256, Aug. 2008.

[9] L. Xu, C. W. Lu, Y. Xu, and J. Jia, "Image smoothing via L0 gradient minimization, "ACM Trans. Graph., vol. 30, no. 6, Dec. 2011,

[10] Art. ID 174. C. C. Pham, S. V. U. Ha, and J. W. Jeon, "Adaptive guided image filtering for sharpness enhancement and noise reduction," in Advances in Image and Video Technology. Berlin, Germany: Springer-Verlag, 2012.

[11]Z. Li, J. Zheng, Z. Zhu, S. Wu, and S. Rahardja, “A bilateral filter in gradient domain," inProc. Int. Conf. Acoust., Speech Signal Process., Mar. 2012, pp. 11131116.

[12] K. He, J. Sun, and X. Tang, "Guided image filtering," IEEE Trans. Pattern Anal. Mach. Intell., vol. 35, no. 6, pp. 1397-1409, Jun. 2013.

[13]Z. Li, J. Zheng, Z. Zhu, S. Wu, W. Yao, and S. Rahardja, "Content adaptive bilateral filtering," in Proc. IEEE Int. Conf. Multimedia Expo, Jul. 2013, pp. 1-6.

[14]Z. Li, J. Zheng, Z. Zhu, and S. Wu, "Selectively detailenhanced fusion of differently exposed images with moving objects, "IEEE Trans. Image Process., vol. 23,no. 10, pp. 4372-4382, Oct. 2014. 